\title{
PIGMENT PRODUCTION BY BACTEROIDES SPECIES WITH REFERENCE TO SUB-CLASSIFICATION
}

\author{
B. I. DUERDEN \\ Department of Bacteriology, Edinburgh University Medical School, \\ Teviot Place, Edinburgh EH8 $9 A G$
}

OlIVER and Wherry (1921) first described bacteroides-like organisms that produced black pigmented colonies when grown on blood agar, and called them Bacterium melaninogenicum. This characteristic appearance was regarded as highly specific and was the sole basis for differentiation from other organisms now generally grouped as Bacteroides. The pigment-producing strains have been studied intermittently since then and much confusion has arisen, partly because of the difficulty experienced by some workers in maintaining them in pure culture (Senos and Mattman, 1955). Some workers were able to study these organisms only in mixed culture and this may be related to a requirement of some strains for vitamin K-like compounds (Lev, 1959). The biochemistry and serology of strains of Bacteroides melaninogenicus was found to vary considerably (Weiss, 1937; Sawyer, MacDonald and Gibbons, 1962), and sub-species have been defined on this basis (Holdeman and Moore, 1972).

Study of the pigment has created more confusion. Oliver and Wherry (1921) thought that it was extracellular and identified it as melanin on the basis of its insolubility in organic solvents and its slow solubility in sodium hydroxide. Schwabacher, Lucas and Rimington (1947) found that the pigment was intracellular and was not melanin but a derivative of haemoglobin; it was spectroscopically identical with haematin.

Tracy (1969) found that clinical isolates of $B$. fragilis produced a dense black pigment in mixed culture with Escherichia coli or Staphylococcus aureus in cooked-meat broth containing a rusty nail. She found that standard strains of $B$. fragilis, B. melaninogenicus and B. necrophorus produced this pigment when grown in pure culture in a medium containing cysteine, ferrous sulphate and vitamin $\mathrm{K}$. The pigment was extracellular and was identified as colloidal ferrous sulphide.

The present series of experiments was undertaken to investigate the nature of the pigment and to study the conditions necessary for its production by Bacteroides, with special reference to its taxonomic significance.

MATERIALS AND METHODS

Throughout these experiments cellular morphology was regularly checked by examination of Gram-stained smears; the purity of cultures was checked by aerobic and anaerobic subculture on human-blood agar. 
Culture inocula. One drop $(0.02 \mathrm{ml})$ of a $48-\mathrm{h}$ cooked-meat broth culture was used to seed each tube and one loopful $(c .0 .01 \mathrm{ml})$ to seed each plate of medium.

Anaerobic incubation. Anaerobic jars supplied by Baird and Tatlock Ltd (BTL) were each fitted with three catalyst sachets and the anaerobic procedure of Collee et al. (1972) was essentially followed. All incubation was at $37^{\circ} \mathrm{C}$ in an atmosphere of $90 \% \mathrm{H}_{2}$ and $10 \% \mathrm{CO}_{2}$.

Organisms. The following strains were obtained from the National Collection of Type Cultures (NCTC): Bacteroides melaninogenicus nos. 9336, 9337 and 9338; B. fragilis no. 9343 ; and $B$. necrophorus nos. 7155 and 10575 . In addition, we studied 36 strains of Gram-negative anaerobic non-sporing bacilli, originally isolated from clinical specimens or human faeces. All these anaerobic bacilli were maintained by fortnightly serial subculture in tubes of cooked-meat broth incubated anaerobically at $37^{\circ} \mathrm{C}$ for $24 \mathrm{~h}$ and thereafter kept on the bench.

Laboratory strains of E. coli, S. aureus (Oxford; no. NCTC6571), Proteus mirabilis and Salmonella typhimurium had been maintained by serial subculture in nutrient broth and on nutrient-agar slopes for many years; a laboratory strain of Clostridium welchii was maintained by serial subculture in cooked-meat broth.

Chemicals. Aqueous solutions of menadione (Sigma, London; $100 \mu \mathrm{g}$ per ml; see Barnes and Impey, 1971); ferrous sulphate (Analar grade; $250 \mathrm{mg}$ per ml); and L-cysteine (Koch-Light, pure; $100 \mathrm{mg}$ per $\mathrm{ml}$ ), were prepared immediately before use. All were sterilised by membrane filtration and added aseptically to (cooled) autoclaved media to give final concentrations of menadione $1 \mu \mathrm{g}$ per $\mathrm{ml}$, ferrous sulphate $5 \mathrm{mg}$ per $\mathrm{ml}$, and cysteine $2 \mathrm{mg}$ per $\mathrm{ml}$.

Media were either freshly prepared or steamed for $30 \mathrm{~min}$. just before use. Media containing menadione or cysteine could not be steamed and were always freshly prepared.

The basic liquid medium was Robertson's cooked-meat broth (Cruickshank, 1969); various combinations of chemicals were added (see text). Horse-blood broth, containing $10 \%$ Defibrinated Horse Blood (Oxoid) in Oxoid Nutrient Broth no. 2, was used to study pigment production in a liquid medium containing blood. Solid media were: (i) human blood-agar containing 5\% human blood in Oxoid Columbia Agar Base; human blood was provided by the Regional Blood Transfusion Service, Edinburgh, and contained $2 \mathrm{~g}$ disodium citrate and $1.7 \mathrm{~g}$ dextrose in $70 \mathrm{ml}$ of water added to $430 \mathrm{ml}$ of whole blood; (ii) lysed humanblood agar prepared by freezing and thawing the blood before incorporating it in the medium; (iii) horse-blood agar (10\% Defibrinated Horse Blood, Oxoid, and Oxoid Columbia Agar Base). Special solid media derived from cooked-meat broth were prepared as follows: (i) the supernate from Robertson's cooked-meat broth solidified with $1 \%$ agar (Oxoid no. 1); and (ii) Robertson's cooked-meat broth made with homogenised meat and solidified with $1 \%$ agar.

\section{Characterisation of strains}

All the NCTC strains were subjected to the following tests. Haemolysis on blood agar was observed after incubation for $48 \mathrm{~h}$ and 1 week. Lipase and phospholipase-C activity were detected by observation of the effects of growth on egg-yolk agar (Cruickshank, 1969). Oxidase production was indicated by a blue colour after a solution of tetramethyl- $p$ phenylene-diamine dihydrochloride had been poured on to cultures on blood agar. Catalase was detected by flooding 1 week's growth on egg yolk agar with $10 \%$ hydrogen peroxide; a stream of bubbles arose from catalase-producing colonies. Gelatinase test: a charcoalgelatin disk (Oxoid) in a cooked-meat broth culture was observed for digestion of the disk during incubation for 2 weeks. Indole production was detected by adding ether followed by a few drops of Ehrlich's Reagent to the liquid phase of a 48-h cooked-meat broth culture; the reagent turned pink in the presence of indole. Aesculin hydrolysis was detected by the development of black discolouration when $0.5 \mathrm{ml}$ of a $1 \%$ aqueous solution of ferric ammonium citrate was added to a 48 -h culture in cooked-meat broth with $1 \%$ aesculin. Production of $\mathrm{H}_{2} \mathrm{~S}$ was detected by blackening of a strip of lead-acetate paper suspended during incubation in the neck of a tube of cooked-meat broth containing cysteine. 
Inhibition of growth by bile salts. Each strain was seeded on to a series of four nutrientagar test media (Oxoid Columbia Agar) supplemented with $1 \%$ sodium succinate and $1 \%$ glucose and containing (i) no bile salt (control); (ii) $0.5 \%$ sodium taurocholate (BDH Chemicals Ltd): (iii) $0.1 \%$ sodium deoxycholate (BDH); or (iv) $0.5 \%$ sodium taurocholate plus $0 \cdot 1 \%$ sodium deoxycholate. The plates were examined for growth after $24 \mathrm{~h}$ and $48 \mathrm{~h}$.

Sugar-fermentation tests. Seitz-filtered aqueous solutions of glucose, lactose, sucrose, and maltose were separately added to cooked-meat broth to give a final concentration of $1 \%$ of the test sugar. These were seeded with $0.02 \mathrm{ml}$ of a $48-\mathrm{h}$ cooked-meat broth culture. Cultures were incubated for 48 hours and the final $p \mathrm{H}$ was measured with a $p \mathrm{H}$ meter after exposure on the bench for at least $1 \mathrm{~h}$. Controls included cultures of the test strains in plain cook-edmeat broth and tubes of the uninoculated $1 \%$ sugar media. The test was regarded as positive if the $p \mathrm{H}$ fell $>0.5$ unit below that of the uninoculated control and $>0.5 p \mathrm{H}$ unit in comparison with the value for the 48-h culture in plain cooked-meat broth (see Rutter, 1970). $p \mathrm{H}$ values were measured by a Pye Ingold combined Glass and Reference Electrode, no. 401(E2), with a Pye Ingold Resistance Thermometer no. 622 as temperature compensator.

Antibiotic-sensitivity tests. All NCTC strains were grown on fresh $5 \%$ human-blood agar and tested for sensitivity to antibiotics by the disk-diffusion method (modified from Sutter and Finegold, 1971). The plates were seeded by spreading $0.02 \mathrm{ml}$ of a 48 -h cookedmeat broth culture on the surface with a standard glass spreader. The sizes of inhibition zones were measured after either 24 or $48 \mathrm{~h}$, as soon as good growth was visible.

Disks containing neomycin sulphate $1000 \mu \mathrm{g}$, kanamycin sulphate $1000 \mu \mathrm{g}$, rifampicin (Rimactane) $10 \mu \mathrm{g}$, and erythromycin ethyl succinate $60 \mu \mathrm{g}$ were prepared in our laboratory. Disks containing lincomycin $2 \mu \mathrm{g}$, and bacitracin 0.1 unit, were supplied by Mast Laboratories Ltd; and disks with colistin sulphate $10 \mu \mathrm{g}$, tetracycline $10 \mu \mathrm{g}$ and penicillin 1.5 units were from Oxoid Ltd.

A standard Oxford staphylococcus was tested in parallel with the Bacteroides spp. as a control. This control organism was incubated anaerobically and the validity of such a control continues to be debated. The control strain was sensitive to neomycin, kanamycin, penicillin, erythromycin, rifampicin, lincomycin, and tetracycline, and resistant to colistin and bacitracin. The tests were performed at least four times and the inhibition-zone diameters were grouped into four grades: $<15 \mathrm{~mm} ; 16-25 \mathrm{~mm} ; 26-35 \mathrm{~mm} ;>35 \mathrm{~mm}$. The grades obtained with the Bacteroides strains were then classified as resistant (R) or sensitive (S) by comparison with the grades obtained with the Oxford staphylococcus.

\section{Isolation and examination of pigment}

Presumptive colloidal ferrous sulphide. The dense black pigment produced by culture of any Bacteroides strain in liquid media containing cysteine and ferrous sulphate was separated from the bacterial cells by centrifugation at $800 \mathrm{~g}$ for $1 \mathrm{~h}$. Most of the pigment remained in the supernate, which retained the dense black appearance of the original culture; some was deposited with the cells. The presence of ferrous ions in the supernate was demonstrated in tests with potassium ferricyanide solution with and without acidification with $\mathrm{HCl}$; the Prussian-blue reaction indicated the presence of ferrous ions.

Lead acetate paper was used to detect the evolution of $\mathrm{H}_{2} \mathrm{~S}$ after acidification of the supernate with $\mathrm{HCl}$. The supernate was also examined by spectrophotometry over the wavelength range $200-700 \mathrm{~nm}$ after 50-fold dilution with distilled water.

Pigment from cultures of $B$. melaninogenicus in $10 \%$ horse blood broth with menadione. The cells were sedimented by centrifugation at $800 \mathrm{~g}$ for $1 \mathrm{~h}$ and the supernate was retained. The deposit of bacterial cells and red-cell debris was washed thrice with distilled water to lyse any remaining $\mathrm{RBC}$ and to remove the haemoglobin; then it was disrupted ultrasonically for $30 \mathrm{~min}$. in distilled water and the cell debris separated from the soluble fraction by ultracentrifugation at $20,000 \mathrm{~g}$ for $1 \mathrm{~h}$. The original culture supernate was diluted 100-fold in distilled water and the aqueous extract after ultrasonication was diluted 10 -fold for spectrophotometry over the wavelength range $200-700 \mathrm{~nm}$. These prepartions were also tested for the presence of sulphide and ferrous ions as above.

Pigment from colonies of $B$. melaninogenicus on blood agar. Growth after anaerobic 
incubation for 1 week on blood agar was suspended in distilled water and treated in the same way as above. The first supernate and the supernate obtained after ultrasonic treatment were examined by spectrophotometry.

Spectrophotometry. A Pye Unicam SP8000 Ultraviolet Recording Spectrophotometer was used.

Ultrasonic disintegration. An MSE-Mullard Ultrasonic Disintegrator no. 4200 was used.

\section{RESULTS}

Growth of Bacteroides spp. on blood agar

B. melaninogenicus strain no. 9338 produced visible colonies on blood agar after anaerobic incubation for $48 \mathrm{~h}$; the colonies became black after anaerobic incubation for 2-4 days. Haemolysis appeared and increased concurrenly with colony pigmentation. The same pattern was observed with strains nos. 9336 and 9337 except that pigmentation and haemolysis did not develop until 4-6 days.

There was essentially no difference between degree and speed of pigmentation on horse-blood agar and human-blood agar. Menadione increased the rate of pigmentation, particularly on horse-blood agar. Pigment appeared earlier on heated (chocolate) horse-blood agar than on unheated horse blood. Pigmentation was most rapid on lysed human-blood agar.

No other Gram-negative anaerobic bacilli produced pigmented colonies in pure culture on human- or horse-blood agar.

Effect of cultivation with Escherichia coli or Staphylococcus aureus (Oxford) on pigment production by Bacteroides spp. on blood agar

The effect of cultivation with $E$. coli or $S$. aureus on pigment production was tested on separate human-blood-agar plates. E. coli or $S$. aureus was seeded on to a plate as a diametric streak and a streak inoculum of each strain of Gram-negative anaerobic bacilli, including the NCTC reference strains, was made at right angles across the $E$. coli or $S$. aureus inoculum so that areas of pure and mixed growth were obtained. After incubation for 1 week, only NCTC strains of $B$. melaninogenicus, nos. 9336, 9337 and 9338, had produced pigmented colonies. Pigment developed more rapidly in strains nos. 9336 and 9337 in areas of mixed culture with E. coli or S. aureus, E. coli stimulating pigment production more than $S$. aureus. No other strains produced pigment.

Growth of Bacteroides spp. in mixed culture with E. coli or S. aureus in cooked meat broth with iron filings

Each strain was incubated separately with $E$. coli and $S$. aureus in cookedmeat broth to which a knife-point of iron filings had been added. After 3 days, one laboratory-isolated strain produced a few small black clumps in mixed culture with $S$. aureus. No others produced pigment.

Growth of Bacteroides spp. with menadione, cysteine and ferrous sulphate

Each strain was grown for $48 \mathrm{~h}$ in plain cooked-meat broth and in cookedmeat broth to which menadione, cysteine, and ferrous sulphate, singly and 
in all combinations, had been added, i.e., eight media in all (see Methods). All produced dense black pigmentation throughout media containing both cysteine and ferrous sulphate; this effect was independent of the presence of menadione. Two strains produced clumps of black pigment in medium with only ferrous sulphate as an additive, and four strains produced black clumps when both ferrous sulphate and menadione were present. Pigment was not produced in media to which ferrous sulphate had not been added.

The same dense black appearance was reproduced by the addition of ferrous sulphate solution to a 48-h culture of any strain in the medium that contained only additional cysteine. The black discolouration began to develop as soon as the ferrous sulphate solution was added. A similar result was obtained when copper sulphate solution was added to 48-h cultures in the medium with additional cysteine; in this case, the precipitate was blue.

\section{Hydrogen-sulphide production in cultures of Bacteroides spp.}

All strains were tested for $\mathrm{H}_{2} \mathrm{~S}$ production in plain cooked-meat broth and in cooked-meat broth with additional cysteine. Weak production of $\mathrm{H}_{2} \mathrm{~S}$ was generally demonstrable with cultures in the plain medium. All strains grown in cooked-meat broth supplemented with cysteine gave strongly positive results after $48 \mathrm{~h}$; the development of the black pigment was correlated with detectable $\mathrm{H}_{2} \mathrm{~S}$ production and the presence of ferrous sulphate.

\section{Studies with bacteria of other genera in fuid media}

Strains of E. coli, $P$. mirabilis, $S$. typhimurium and $C$. welchii were also inoculated separately into the above test media. They all gave a strongly positive test for $\mathrm{H}_{2} \mathrm{~S}$ production in media containing added cysteine and they produced the same dense black pigment throughout the medium when ferrous sulphate was also present. The naked-eye appearances of the black broth cultures were indistinguishable from those produced by the Bacteroides strains.

\section{Growth of Bacteroides spp. on solid media containing cysteine, menadione and ferrous sulphate}

Ten strains were incubated on human-blood agar with cysteine and ferrous sulphate incorporated in the medium and with disks of menadione $(0.005 \mathrm{mg})$ and phytomenadione $(0.1 \mathrm{mg}$ and $0.005 \mathrm{mg})$ on the surface. One strain failed to grow; the others produced poor growth and colonies were not pigmented.

All strains were incubated on separate agar media incorporating (i) cookedmeat-broth supernate, (ii) homogenised cooked meat, or (iii) lysed human blood, each containing cysteine, menadione and ferrous sulphate. Fourteen failed to grow on the supernate agar and the remainder produced barely visible growth without pigmentation. All except two strains grew on homogenised cooked-meat-broth agar, but growth was slow and colonies were small and not pigmented. Eight strains grew moderately well on lysed-blood agar; 14 produced barely visible growth and 23 failed to grow. After 1 week, 
colonies of the eight moderately good cultures began to develop black centres and after 10 days most colonies had black centres. None of these strains was $B$. melaninogenicus and one was a known $B$. fragilis strain no. 9343.

Examination of black pigment from cultures of Bacteroides strains and E. coli in cooked-meat broth with cysteine and ferrous sulphate

The black pigment did not sediment on centrifugation, and a wet film of the deposit showed normal bacterial cells with clumps of extracellular pigment. The cell-free, pigmented supernate did not react with potassium ferricyanide solution; this indicated the absence of free ferrous ions, whereas the supernate from the medium control gave a strong Prussian-blue reaction attributable to the ferrous sulphate content. When the culture supernate was acidified with $\mathrm{HCl}, \mathrm{H}_{2} \mathrm{~S}$ was evolved and the pigmentation gradually cleared. The acidified supernate gave a strongly positive Prussian-blue reaction with potassium ferricyanide solution. This is prima-facie evidence that this pigment was the colloidal ferrous sulphide identified by Tracy (1969) in similar circumstances. Spectrophotometry of a 50 -fold dilution of the black supernate, the medium control and plain cooked-meat broth revealed a general increase in turbidity over the whole range for the black supernate as one might expect with a black substance in colloidal suspension.

\section{Examination of pigment produced by $B$. melaninogenicus in blood-containing media}

B. melaninogenicus strains nos. 9336, 9337 and 9338, B. fragilis strain no. 9343 and $B$. necrophorus strains nos. 7155 and 10575 were grown for 1 week in horse-blood broth with menadione. The centrifuged culture supernate and an aqueous ultrasonic extract of the deposited bacterial cells were examined by spectrophotometry for pigmentation. There was no difference between the spectra of the supernates from the six cultures and from an uninoculated control. There was a small increase in absorption at $525-575 \mathrm{~nm}$ and a single large narrow peak at $405-410 \mathrm{~nm}$ representing haemoglobin from lysed RBC. When the cells were washed with distilled water, haemoglobin was again the only pigment detected in the washings and it was equally present in all six cultures and the control. The final washings were clear.

At this stage the cells of $B$. melaninogenicus strains were considerably darker than those from the test cultures of $B$. fragilis and $B$. necrophorus strains. The pigment was clearly cell-associated and not freely extracellular. The cells were disrupted ultrasonically in distilled water and the supernate, after ultracentrifugation to remove cell debris, was dark brown in the case of $B$. melaninogenicus strains and colourless in the case of $B$. fragilis and $B$. necrophorus strains and the medium control. Spectrophotometry of these aqueous extracts from the disrupted cells showed a broad peak in the $370-450 \mathrm{~nm}$ band, with a maximum at $410 \mathrm{~nm}$, for $B$. melaninogenicus strains; this was not so with preparations derived from $B$. fragilis or $B$. necrophorus strains or from the medium control (fig. 1). 


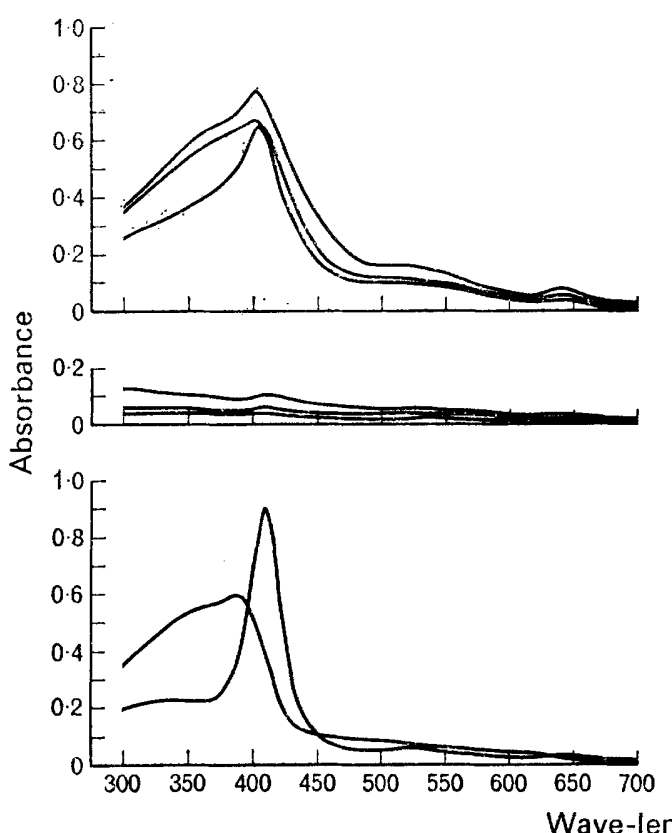

FIG. 1.-Typical patterns of plot obtained in spectrophotometric studies on extracts of disintegrated washed preparations of Bacteroides species derived from cultures in horse-blood broth: top graph: $B$. melaninogenicus strains nos. 9336, 9337 and 9338; middle graph: $B$. fragilis strain no. 9343 and $B$. necrophorus strains nos. 7155 and 10575 ; bottom graph: aqueous solution of haemin (broader peak) and aqueous extract of lysed horse red cells (narrow peak).
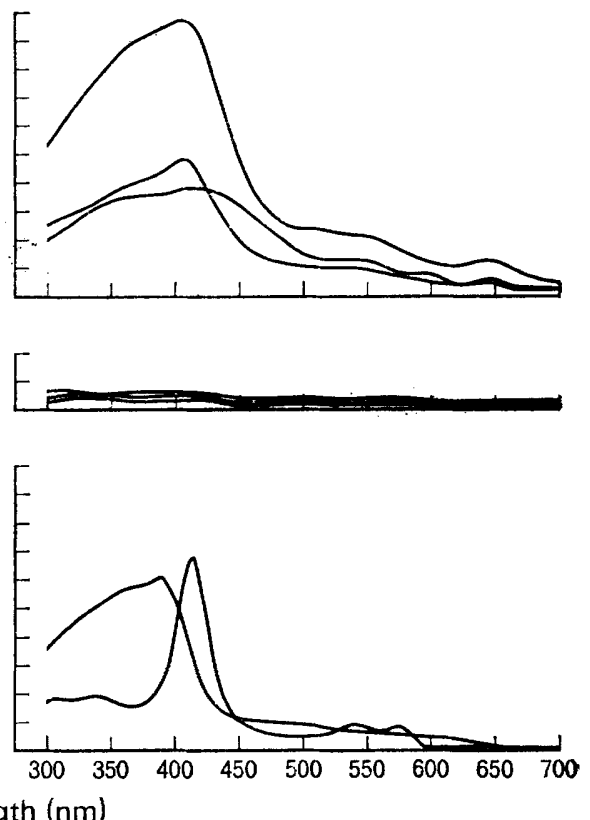

FIG. 2.-Typical patterns of plots obtained in spectrophotometric studies on extracts of disintegrated washed preparations of $\mathrm{BaC}$ teroides species derived from cultures on human-blood agar: top graph: $B$. melaninogenicus strains nos. 9336, 9337 and 9338 (upper line); middle graph: $B$. fragilis strain no. 9343 and $B$. necrophorus strains nos. 7155 and 10575 ; bottom graph: aqueous solution of haemin (broader peak) and aqueous extract of lysed human red cells (narrow peak.)

The Prussian-blue reaction was not produced by the addition of potassium ferricyanide solution to this pigment extract, either with or without acidification, and $\mathrm{H}_{2} \mathrm{~S}$ was not evolved after acidification.

Colonies of $B$. melaninogenicus strains nos. 9336, 9337 and $9338, B$. fragilis strain no. 9343 and B. necrophorus strains nos. 7155 and 10575 after incubation for 1 week on blood agar were suspended in distilled water, washed and examined as above. The first washing showed a small amount of haemoglobin carried over with each, but subsequent washings were clear. The $B$. melaninogenicus cells remained dark and the $B$. fragilis and $B$. necrophorus cells were pale. After ultrasonic treatment and ultracentrifugation, the extracts from $B$. melaninogenicus strains were all dark brown and showed spectrophotometry peaks very similar to those obtained from cells grown in horse-blood broth. The extracts from $B$. fragilis and $B$. necrophorus strains had no such peak (fig. 2).

\section{Characterisation of NCTC strains}

All of the test strains were non-motile, non-sporing, pleomorphic, Gramnegative and obligately anaerobic bacteria. Gram-stained films of B. melanino- 
genicus strains nos. 9336, 9337 and 9338 showed short bacilli and cocco-bacilli, often in pairs and forming some short chains; $B$. fragilis strain no. 9343 consisted of highly pleomorphic bacilli with some short filaments but no chains; $B$. necrophorus strain no. 10575 consisted of pleomorphic bacilli with many long filaments and some long chains, particularly in preparations from cooked-meat-broth cultures, but strain no. 7155 had a much more uniform appearance of medium-sized bacilli with a few short chains.

The results of the biochemical tests on these strains are shown in table $\mathbf{I}$. The three $B$. melaninogenicus strains were morphologically and biochemically similar except that strain no. 9337 was non-saccharolytic whereas strains nos. 9336 and 9338 fermented glucose, sucrose and maltose but not lactose; strain no. 9338 also produced a lipase and a phospholipase effect on egg-yolk agar. They were clearly different from the following three test strains. $B$. fragilis strain no. 9343 and $B$. necrophorus strains nos. 7155 and 10575 were distinguished from each other by these results; in particular, strain no. 7155 was clearly different from strain no. 10575.

TABLE I

Results of biochemical tests with reference strains of Bacteroides spp

\begin{tabular}{|c|c|c|c|c|c|c|}
\hline \multirow{2}{*}{ Test } & \multicolumn{6}{|c|}{ Results obtained with strain no.* } \\
\hline & 9336 & 9337 & 9338 & 9343 & 7155 & 10575 \\
\hline Aerobic growth $\dagger$ & - & - & - & - & - & - \\
\hline Motility & - & - & - & - & - & - \\
\hline Black pigment on blood agar & + & + & + & - & - & - \\
\hline Haemolysis on blood agar & + & + & + & - & - & + \\
\hline Pearly layer on EYA (lipase) & - & - & + & - & - & + \\
\hline Phospholipase production & - & - & + & - & - & + \\
\hline Oxidase production & - & - & - & - & - & - \\
\hline Catalase production & - & - & - & - & - & - \\
\hline $\mathrm{H}_{2} \mathrm{~S}$ production & + & + & + & + & + & + \\
\hline Indole production & + & + & + & - & - & $i$ \\
\hline Digestion of gelatin & + & $t$ & + & - & - & - \\
\hline Hydrolysis of aesculin & - & - & - & + & + & - \\
\hline $\begin{array}{l}\text { Inhibition of growth by: } \\
0.5 \% \text { sodium taurocholate }\end{array}$ & & & & & & \\
\hline $\begin{array}{l}0.5 \% \text { sodium taurocholate } \\
0.1 \% \text { sodium deoxycholate }\end{array}$ & I & I & I & $\rightarrow$ & - & I \\
\hline $\begin{array}{l}0.1 \% \text { sodium deoxycholate } \\
0.5 \% \text { sodium taurocholate }\end{array}$ & I & I & I & I & - & I \\
\hline $\begin{array}{l}+0 \cdot 1 \% \text { sodium deoxy- } \\
\text { cholate }\end{array}$ & $\mathbf{I}$ & I & $\mathbf{I}$ & - & - & I \\
\hline Fermentation of: & & & & & & \\
\hline glucose & + & - & + & + & + & - \\
\hline lactose & - & . & - & + & + & - \\
\hline sucrose & + & - & + & + & + & - \\
\hline maltose & + & - & + & + & + & - \\
\hline
\end{tabular}

* B. melaninogenicus NCTC nos. 9336, 9337, and 9338; $B$. fragilis NCTC no. 9343; B. necrophorus NCTC nos. 7155 and $10575 ;+=$ positive result; $-=$ negative result; $I=$ inhibition of growth by the bile salts.

$\dagger$ Aerobic incubation was at $37^{\circ} \mathrm{C}$ (i) in air and (ii) in air with $10 \% \mathrm{CO}_{2}$ added. 


\section{Antibiotic-disk sensitivity tests}

The results of these tests are shown in table II. In general, different antibiograms were obtained with the $B$. fragilis strain no. 9343, the $B$. melaninogenicus strains, and the $B$. necrophorus strains. The three $B$. melaninogenicus strains had similar antibiotic-sensitivity patterns except that strain no. 9337 was resistant to colistin; they were sensitive to all of the other antibiotics except kanamycin (1000- $\mu \mathrm{g}$ disk). The only difference between strains no. 7155 and 10575 was that strain no. 7155 was resistant to erythromycin $(60-\mu \mathrm{g}$ disk).

\section{DISCUSSION}

The importance of Gram-negative anaerobic non-sporing bacteria of the Bacteroides group in clinical medicine is being increasingly recognised, but the development of precise methods for their identification and differentiation remains a major problem. In particular, the confusion that has surrounded studies on B. melaninogenicus, because of the difficulties experienced in maintaining pure cultures, the variability of results of biochemical tests, and the specific growth requirements of some strains (Gibbons and MacDonald, 1960), has resulted in the distinctive property of pigmentation remaining the primary basis for the identification of $B$. melaninogenicus. Bacteroides-like organisms that produce black colonies on blood agar have been grouped together as B. melaninogenicus by some workers solely on the basis of this property, irrespective of any other similarities or differences (Beerens, 1970). Doubt was cast on the validity of this approach by the claim of Tracy (1969) that other Bacteroides species can produce black pigment under particular cultural conditions, and that the pigment is extracellular colloidal ferrous sulphide. Earlier workers had identified the pigment of $B$. melaninogenicus as extra-

TABLE II

Results of antibiotic-disk-sensitivity tests with reference strains of Bacteroides spp.

\begin{tabular}{|c|c|c|c|c|c|c|c|}
\hline \multirow{2}{*}{$\begin{array}{c}\text { Antibiotic } \\
\text { (content per disk) }\end{array}$} & \multicolumn{7}{|c|}{ Sensitivity or resistance of strain no.* } \\
\hline & 9336 & 9337 & 9338 & 9343 & 7155 & 10575 & $\begin{array}{l}\text { S. aureus } \\
\text { (Oxford) }\end{array}$ \\
\hline $\begin{array}{l}\text { Neomycin }(1000 \mu \mathrm{g}) \\
\text { Kanamycin }(1000 \mu \mathrm{g}) \\
\text { Penicillin }(1 \cdot 5 \mathrm{unit}) \\
\text { Erythromycin }(60 \mu \mathrm{g}) \\
\text { Colistin }(10 \mu \mathrm{g}) \\
\text { Rifampicin }(10 \mu \mathrm{g}) \\
\text { Lincomycin }(2 \mu \mathrm{g}) \\
\text { Bacitracin }(0 \cdot 1 \mathrm{unit}) \\
\text { Tetracycline }(10 \mu \mathrm{g})\end{array}$ & $\begin{array}{l}\mathbf{S} \\
\mathbf{R} \\
\mathbf{S} \\
\mathbf{S} \\
\mathbf{S} \\
\mathbf{S} \\
\mathbf{S} \\
\mathbf{S} \\
\mathbf{S}\end{array}$ & $\begin{array}{l}\text { S } \\
\mathbf{R} \\
\mathbf{S} \\
\mathbf{S} \\
\mathbf{R} \\
\mathbf{S} \\
\mathbf{S} \\
\mathbf{S} \\
\mathrm{S}\end{array}$ & $\begin{array}{l}\mathrm{S} \\
\mathrm{R} \\
\mathrm{S} \\
\mathrm{S} \\
\mathrm{S} \\
\mathrm{S} \\
\mathrm{S} \\
\mathrm{S} \\
\mathrm{S}\end{array}$ & $\begin{array}{l}\mathbf{R} \\
\mathbf{R} \\
\mathbf{R} \\
\mathbf{S} \\
\mathbf{R} \\
\mathrm{S} \\
\mathbf{R} \\
\mathrm{R} \\
\mathrm{S}\end{array}$ & $\begin{array}{l}\mathrm{S} \\
\mathbf{S} \\
\mathbf{S} \\
\mathbf{R} \\
\mathbf{S} \\
\mathbf{R} \\
\mathrm{S} \\
\mathrm{R} \\
\mathrm{S}\end{array}$ & $\begin{array}{l}\mathbf{S} \\
\mathbf{S} \\
\mathbf{S} \\
\mathbf{S} \\
\mathbf{S} \\
\mathbf{R} \\
\mathbf{S} \\
\mathbf{R} \\
\mathbf{S}\end{array}$ & $\begin{array}{l}\mathbf{S} \\
\mathbf{S} \\
\mathbf{S} \\
\mathbf{S} \\
\mathbf{R} \\
\mathbf{S} \\
\mathbf{S} \\
\mathbf{R} \\
\mathrm{S}\end{array}$ \\
\hline
\end{tabular}

* B. melaninogenicus NCTC nos. 9336, 9337 and $9338 ;$ B. fragilis NCTC no. 9343; B. necrophorus NCTC nos. 7155 and 10575 . $\mathrm{S}=$ Sensitive; $\mathrm{R}=$ resistant (see Methods). 
cellular melanin (Oliver and Wherry, 1921), whereas Schwabacher et al. (1947) considered that it was intracellular or cell-associated haematin.

The first group of experiments in the present study with 42 bacteroides-like organisms and one strain of $E$. coli, $P$. mirabilis, $S$. typhimurium and $C$. welchii grown in media containing additional cysteine and ferrous sulphate showed that all of these organisms produce $\mathrm{H}_{2} \mathrm{~S}$ in such a medium and all produce a dense black colloidal precipitate of ferrous sulphide. Tracy (1969) regarded this as a true bacterial extracellular pigment synthesised by all species of Bacteroides under appropriate conditions; she argued that all could then be called " $B$. melaninogenicus" and hence sought to explain the variability in biochemical reactions of different strains of " $B$. melaninogenicus". However, the present results show that the production of ferrous sulphide in this way is not equivalent to the synthesis of a specific pigment; it is an inorganic chemical reaction occurring whenever $\mathrm{H}_{2} \mathrm{~S}$ is introduced into a solution containing ferrous ions. When a sulphur source such as cysteine was provided, all the Bacteroides-like organisms produced $\mathrm{H}_{2} \mathrm{~S}$; ferrous sulphate, either incorporated in the original medium or added after incubation, was then reduced to ferrous sulphide and merely acted as an indicator of $\mathrm{H}_{2} \mathrm{~S}$ production. This indicator system, however, is not responsible for the development of the typical black colonies that form when Bacteroides strains called B. melaninogenicus are grown on media containing blood.

In the second part of the present investigation, three reference strains of B. melaninogenicus were grown in media containing blood, and the washed bacterial cells and the cell-free medium supernate were examined for pigment. There was no extracellular pigment in the supernate, and pigment was not eluted when the cells were washed with distilled water, but the washed cells of the three B. melaninogenicus strains were dark brown in colour. Parallel treatment of cultures of $B$. fragilis and $B$. necrophorus yielded pale cells. The dark pigment that gives the colonies of $B$. melaninogenicus their characteristic appearance is either intracellular or cell-associated in such a way that it cannot be removed by washing with water despite the finding that, when released by ultrasonic treatment of the cells, the pigment is water soluble and can then be extracted in aqueous solution.

The extracted pigment has none of the properties of ferrous sulphide; it is in solution and not in suspension; it does not give the Prussian-blue reaction with potassium ferricyanide solution after acidification; and acidtreatment does not liberate $\mathrm{H}_{2} \mathrm{~S}$ from it. Moreover, ferrous sulphide produced in colonies of other bacteroides-like organisms grown on lysed-blood agar with additional cysteine and ferrous sulphate faded when left exposed to air and light; the (intact) pigmented colonies of B. melaninogenicus did not fade. When the pigment was examined by spectrophotometry, there was an absorption peak at $410 \mathrm{~nm}$. This is the wavelength at which haemoglobin in the medium supernate gives its peak. The position of the peak is constant for extracts from all three $B$. melaninogenicus strains grown in blood broth or on blood agar, whereas a comparable peak was not given by extracts from $B$. fragilis or $B$. necrophorus. Moreover, the likely source of the pigment is indicated by the 
observations that pigmentation develops only in colonies on blood agar in association with haemolysis of the surrounding medium and is more rapid when lysed blood is used for the medium. These results support the conclusions of Schwabacher et al. (1947) that the pigment of B. melaninogenicus is derived from haemoglobin and is either intracellular or cell-associated.

The present experiments and those of Schwabacher et al. are open to the criticism that the pigment was extracted from preparations of cells grown in blood-containing media. In both studies, however, great care was taken to wash away all traces of extraneous haemoglobin; and in the present study no trace of haemoglobin was revealed by spectrophotometry in control extracts similarly derived from cultures of $B$. fragilis and B. necrophorus or in control extracts of the uninoculated medium.

These results indicate that pigment production during growth in bloodcontaining media is a stable characteristic of certain strains of Bacteroides, at present all called $B$. melaninogenicus. It is not a property that can be expressed by any strain of Bacteroides under suitably manipulated cultural conditions. However, it does not follow that all pigment-producing strains form a biochemically homogeneous group: the three pigment-producing strains used in the present study are not identical. The results of our biochemical tests and antibiotic-disk-sensitivity tests show that strain no. 9337 is clearly different from the other two (see tables I and II); it is non-saccharolytic, resistant to colistin and does not produce a lipase or phospholipase. However, the three pigment-producing strains have more properties in common with each other than any one of them has with a strain of $B$. fragilis or $B$. necrophorus. The three species were differentiated on the basis of pigmentation, digestion of gelatin, indole production, aesculin hydrolysis, bile-salt sensitivity and antibiogram. Furthermore, $B$. melaninogenicus strains were culturally more demanding than $B$. fragilis and $B$. necrophorus strains. Cooked-meat-broth cultures of $B$. melaninogenicus held on the bench died more rapidly; subculture at 10-12-day intervals was needed to ensure survival of $B$. melaninogenicus, whereas $B$. fragilis and $B$. necrophorus strains survived for at least 14 days. It is also significant that the morphological and biochemical differences between $B$. necrophorus strains nos. 7155 and 10575 are so considerable that the two strains cannot reasonably be regarded as belonging to the same species (Collee and Watt, personal communication).

From the present study it appears that pigmentation on blood-containing media is a significant property and merits considerable weight in the taxonomy of the Bacteroides group. It is, however, only one feature and it must be clear that there can be wide variations between pigment-producing strains in their biochemical properties and antibiograms. Some workers have described pigmented Bacteroides strains that varied in their individual growth requirements (Lev, 1959; Gibbons and MacDonald, 1960). Other workers have described pigmented Bacteroides strains that have different biochemical properties (Sawyer et al., 1962; Holdeman and Moore, 1972) and antibiograms (Finegold, Harada and Miller, 1967) and have identified sub-species of $B$. melaninogenicus on these grounds. They retain the species name $B$. melanino- 
genicus for all pigment-producing strains, even when pigmentation may be the only significant shared characteristic. Provided that such widely different subspecies of a single species are acceptable, it is reasonable to regard all pigmented strains of Bacteroides as belonging to the species $B$. melaninogenicus and then divide that into sub-species. The problem then arises that it could be expected that strains of Bacteroides would be described that were identical with a subspecies of $B$. melaninogenicus in biochemical properties and antibiogram but did not produce pigment. The identification of such strains would depend upon future decisions based upon the biochemical investigations and antibiogram studies of Bacteroides spp. being undertaken in several centres. Finegold et al. (1967) advocated a scheme of identification based upon antibiograms and this appears to differentiate well between the species when high-concentration disks of neomycin $(1000 \mu \mathrm{g})$, kanamycin $(1000 \mu \mathrm{g})$, and erythromycin $(60 \mu \mathrm{g})$ (Sutter and Finegold, 1971) are used in addition to the commonly used disks of pencillin (1.5 units), rifampicin $(10 \mu \mathrm{g})$, colistin $(10 \mu \mathrm{g})$ and bacitracin $(0 \cdot 1$ unit). As a long-term approach to identification this method carries the risk of invalidation by acquired drug resistance and more experience is needed for its evaluation.

Other groups (Werner, Pulverer and Reichertz, 1971; Holdeman and Moore, 1972) have concentrated upon gas-liquid chromatography to detect the acid end-products of glucose fermentation and used these results to differentiate between the Bacteroides spp. It remains to be decided whether pigmentation remains the significant basis of identification of $B$. melaninogenicus or whether antibiograms, gas-liquid chromatography of the acid end-products of glucose fermentation, or some other group of biochemical tests, will provide more valid criteria for the taxonomy of the Bacteroides group.

\section{SUMMARY}

All of six reference strains of Bacteroides species, 36 laboratory isolates conforming to this group, and individual strains of Escherichia coli, Proteus mirabilis, Salmonella typhimurium and Clostridium welchii produced a dense black pigment, identified as ferrous sulphide, when grown in cooked-meat media containing cysteine and ferrous sulphate. This was an indicator effect resulting from the production of $\mathrm{H}_{2} \mathrm{~S}$ by the bacteria in the presence of ferrous ions and was unrelated to the characteristic pigment produced by strains of $B$. melaninogenicus when grown on blood agar. A pigment was extracted by ultrasonic disintegration of washed cells of three reference strains of B.melaninogenicus grown for 1 week in horse-blood broth and on human-blood agar. It was intracellular or cell-associated, soluble in water and had the spectrophotometric characteristics of a derivative of haemoglobin. No such pigment was extracted from strains of $B$. fragilis or $B$. necrophorus by similar procedures. Pigment production is a stable characteristic of those strains of Bacteroides called B. melaninogenicus and it is a significant property in the classification of the Bacteroides group. However, the pigment-producing strains are not a homogeneous species, and there were considerable differences between the 
results of biochemical tests and antibiograms obtained with the three strains of $B$. melaninogenicus.

I wish to thank Dr J. G. Collee for much helpful advice and encouragement, and Mr R. Brown for his skilled technical assistance. Dr Collee acknowledges a valuable discussion with Dr Ella Barnes. The financial support of the Medical Research Council (Grant G971/115/B) is gratefully acknowledged.

\section{REFERENCES}

BARNES, E. M. AND IMPEY, C. S. 1971. The isolation of the anaerobic bacteria from chicken caeca with particular reference to members of the family Bacteroidaceae. In Isolation of anaerobes, (Soc. Appl. Bact. Tech. Series No. 5), edited by D. A. Shapton and R. G. Board, London and New York, p. 118.

BeERENS, H. 1970. Report of the International Committee on Nomenclature of Bacteria Taxonomic Subcommittee for Gram-negative Anaerobic Rods. Int. J. syst. Bact., 20, 297.

Collee, J. G., Watt, B., Fowler, E. B. AND Brown, R. 1972. An evaluation of the Gaspak system in the culture of anaerobic bacteria. J. appl. Bact., 35, 71.

Cruickshank, R. 1969. Medical microbiology, 11th ed. (revised reprint), Edinburgh and London, p. 757.

Finegold, S. M., Harada, N. E. AND Miller, L. G. 1967. Antibiotic susceptibility patterns as aids in classification and characterisation of Gram-negative anaerobic bacilli. J. Bact., 94, 1443.

GibBons, R. J. AND MaCDonald, J. B. 1960. Hemin and vitamin K compounds as required factors for the cultivation of certain strains of Bacteroides melaninogenicus. J. Bact., $80,164$.

HOLDEMAN, L. V. AND MOORE, W. E. C. 1972. Anaerobe laboratory manual. Blacksburg, Va., p. 27.

LEV, M. 1959. The growth promoting activity of compounds of the vitamin $\mathrm{K}$ group and analogues for a rumen strain of Fusiformis nigrescens (Bact. melaninogenicum). J. gen. Microbiol., 20, 697.

OLIVER, W. W. AND WHERRY, W. B. 1921. Notes on some bacterial parasites of the human mucous membranes. J. infect. Dis., 28, 341.

RUTTER, J. M. 1970. A study of the carbohydrate fermentation reactions of Clostridium oedematiens (Cl. novyi). J. med. Microbiol., 3, 283.

SAwyer, S. J., MacDonald, J. B. ANd GibBons, R. J. 1962. Biochemical characteristics of Bacteroides melaninogenicus. A study of thirty-one strains. Archs oral Biol., 7, 685 .

SChWABACHER, H., LuCAS, D. R. AND Rimington, C. 1947. Bacterium melaninogenicusa misnomer. J. gen. Microbiol., 1, 109.

Senos, G. AND Mattman, L. H. 1955. Notes on Bacteroides melaninogenicus. J. Bact., 70, 483.

SutTer, V. L. AND Finegold, S. M. 1971. Antibiotic disc susceptibility testing for rapid presumptive identification of Gram-negative anaerobic bacilli. Appl. Microbiol., 21, 13.

Tracy, O. 1969. Pigment production in Bacteroides. J. med. Microbiol., 2, 309.

WeIss, C. 1937. Observations on Bacterium melaninogenicum: demonstration of fibrinolysin, pathogenicity and serological types. Proc. Soc. exp. Biol. Med., 37, 473.

Werner, H., Pulverer, G. AND Reichertz, C. 1971. The biochemical properties and antibiotic susceptibility of Bacteroides melaninogenicus. Med. Microbiol. Immunol., Berlin, 157, 3. 